\title{
Mink S38G Gene Polymorphism and Atrial Fibrillation in the Chinese Population: A Meta-Analysis of 1871 Participants
}

\author{
Yan-yan Li, ${ }^{1}$ Lian-sheng Wang, ${ }^{2}$ and Xin-zheng $\mathrm{Lu}^{2}$ \\ ${ }^{1}$ Department of Geriatrics, First Affiliated Hospital of Nanjing Medical University, Nanjing 210029, China \\ ${ }^{2}$ Department of Cardiology, First Affiliated Hospital of Nanjing Medical University, Nanjing 210029, China \\ Correspondence should be addressed to Yan-yan Li; lyynjmu123@126.com
}

Received 13 November 2013; Accepted 5 January 2014; Published 16 February 2014

Academic Editors: M. Fontès and A. Latorre

Copyright (c) 2014 Yan-yan Li et al. This is an open access article distributed under the Creative Commons Attribution License, which permits unrestricted use, distribution, and reproduction in any medium, provided the original work is properly cited.

\begin{abstract}
Mink gene S38G polymorphism in the $\beta$-subunit of slow activating component of the delayed rectifier potassium channel current potassium channel has been associated with increased atrial fibrillation (AF) risk. However, the individual studies results were still controversial. To investigate the association of Mink S38G gene polymorphisms with AF, a meta-analysis including 1871 subjects from six individual studies was conducted. Mink S38G gene polymorphism was significantly related to AF under allelic (OR: 1.380, 95\% CI: 1.200-1.600, $P<0.00001$ ), recessive (OR: 1.193, 95\% CI: 1.033-1.377, $P=0.017$ ), dominant (OR: 1.057, 95\% CI: $1.025-1.089, P<0.00001$ ), additive (OR: 1.105 , 95\% CI: 1.036-1.178, $P=0.002$ ), homozygous (OR: 1.128, 95\% CI: $1.068-1.191$, $P<0.00001$ ), and heterozygous genetic models (OR: 1.078, 95\% CI: 1.014-1.146, $P=0.016$ ). A significant association between Mink S38G gene polymorphism and AF risk was found. G allele carriers may predispose to AF.
\end{abstract}

\section{Introduction}

Atrial fibrillation (AF) is the most common arrhythmia in clinical practice [1]. Its prevalence varies among countries, with different ranges in community- and hospital-based studies $(0.1-4 \%$ and $2.8-14 \%$, resp.). The incidence of AF increases with age, ranging from $1 \%$ in youth to approximately $10 \%$ above the age of 80 [2]. AF can cause serious complications such as stroke and congestive heart failure [3, 4]; however, its pathogenesis is still unclear. Electricity remodeling has an important effect on the AF process [5]. The electrophysiologic basis of electricity remodeling is characterized by ion channel dysfunction as the intracellular calcium overloads and the internal flow of L-type calcium channel decreases [6]. The increase of the slow activating component of the delayed rectifier potassium channel current (Iks) can relatively shorten the phase 2 plateau of the action potential and the refractory period which lead to AF occurrence. The myocardium Iks $\beta$-subunit gene (KCNE1, Mink) S38G gene polymorphism has been associated with Iks function enhancement [7].

Mink gene is located in 21q22.1-22.2, spans about 390 bp, encodes 130 amino acids, and constitutes the Iks $\beta$-subunit. Lai et al. (2002) found that a Mink gene rs1805127 locus at the 112th base adenine (A) was substituted with a guanine $(G)$ at the exon region, resulting in the wild-type Serine (Ser, S) being replaced by Glycine (Gly, G) at 38th amino acid in the 3rd exon. They also found that the Mink S38G gene polymorphism was correlated with $\mathrm{AF}$ [7].

Several studies have associated Mink S38G gene polymorphism with AF; however, the results were contradictory. Zeng et al. (2005) found that Mink S38G gene polymorphism was not associated with AF in a study conducted with Beijing Chinese population [8]. By contrast, $\mathrm{Qu}$ and Yin found that Mink 38G allele was significantly correlated with AF in a study conducted with Chongqing Chinese population [9].

In the current study, a meta-analysis that includes $856 \mathrm{AF}$ patients and 1015 controls was conducted to determine the relationship of Mink S38G gene polymorphism with AF in the Chinese population.

\section{Materials and Methods}

2.1. Publication Search and Inclusion Criteria. The terms "Mink," "S38G," "KCNE1," "atrial fibrillation," and "polymorphism" were searched in the electronic databases of PubMed, Embase, Web of Science, China Biological Medicine 
TABLE 1: Characteristics of the investigated studies of the association of Mink S38G gene polymorphism and atrial fibrillation (AF) in the Chinese population.

\begin{tabular}{|c|c|c|c|c|c|c|c|c|c|}
\hline \multirow{2}{*}{ Author } & \multirow{2}{*}{ Year } & \multicolumn{3}{|c|}{$\mathrm{AF}$} & \multicolumn{3}{|c|}{ Control } & \multirow{2}{*}{ Matching criteria } & \multirow{2}{*}{ Sample size (AF/control) } \\
\hline & & SS & SG & GG & SS & SG & GG & & \\
\hline Lai et al. [7] & 2002 & 7 & 37 & 64 & 18 & 44 & 46 & Age, sex, BMI, ethnicity & $108 / 108$ \\
\hline Ni et al. [10] & 2004 & 3 & 37 & 54 & 10 & 48 & 72 & Age, sex, ethnicity & $94 / 130$ \\
\hline Zeng et al. [8] & 2005 & 10 & 60 & 71 & 23 & 100 & 115 & Age, sex, ethnicity & $141 / 238$ \\
\hline Qu and Yin [9] & 2008 & 6 & 26 & 31 & 15 & 32 & 17 & Age, sex, ethnicity & $63 / 64$ \\
\hline Xu et al. [11] & 2008 & 9 & 61 & 77 & 16 & 56 & 75 & Age, sex, BMI, ethnicity & $147 / 147$ \\
\hline Yao et al. [12] & 2011 & 28 & 117 & 158 & 40 & 159 & 129 & Age, sex, BMI, ethnicity & $303 / 328$ \\
\hline
\end{tabular}

AF: atrial fibrillation; BMI: body mass index.

Polymerase chain reaction-restriction fragment length polymorphism genotyping method and case-control study design have been adopted in all of the above studies.

Database, and China National Knowledge Infrastructure. The last updated research date was November 12, 2013, and the publication years ranged from 2002 to 2011.

The selected studies have to be consistent with the following major criteria: (a) assessment of the Mink S38G gene polymorphism and nonvalvular AF, studies on the family history of AF or valvular AF would be excluded; (b) AF was diagnosed as the episodes $\geq 2$ occasions ( $>6$ months apart) by serial 12-lead electrocardiography (ECG) or $24 \mathrm{~h}$ Holter monitoring. The ECG diagnosis criteria were as follows: (1) $P$ wave disappearance and replacement with irregular baseline fluctuation, with a frequency ranging from 350 per minute to 600 per minute, namely, $f$ wave; (2) extremely irregular ventricular rate; (3) inconsistent QRS complex morphology, the QRS complex becomes broader and changes in the presence of aberrant ventricular conduction and the RR internal remains extremely irregular; (c) the study should be a case-control or cohort study published in an official journal; (d) the study should conform to the Hardy-Weinberg equilibrium (HWE).

2.2. Data Extraction. The data were extracted according to a standard protocol. Three investigators carried out the metaanalysis; two of whom searched for parallel studies, and the third served as the arbitrator in case of discordance between the two investigators. Studies that failed to meet the inclusion criteria, were published repeatedly, or provided insufficient data were excluded. Similar data from different studies were only used once. The abstracted data contained the following: the first author's name, publication year, study region, PubMed identifier number, study design, case selection method, matching factors (if applicable), source population, mean ages of the cases and controls, sample size, variants, major and minor alleles, genotype counts for the cases and controls, HWE among controls, and genotyping methods used. The six selected studies were scored based on the criteria implemented from published recommendations on the evaluation of the quality of genetic association studies [13].

2.3. Statistical Analyses. In the present meta-analysis, six genetic models, including the allelic (distribution of $\mathrm{G}$ allelic frequency of Mink S38G gene polymorphism), recessive
( $G G$ versus SG + SS), dominant ( GG + SG versus SS), additive ( $G$ versus $S$ ), homozygous (GG versus SS), and heterozygous genetic models (SG versus SS), were adopted. The relationship of Mink S38G gene polymorphism with AF was compared using odds ratio (OR) and its corresponding 95\% confidence interval (CI). The Chi-square-based Q-tests were used to calculate the heterogeneity among the individual studies, with significance level at $P<0.10$ [14]. The randomeffect model was used to estimate the pooled OR (DerSimonian and Laird methods) when heterogeneity existed among the individual studies [15]. Otherwise, the fixed-effect model was used (the Mantel-Haenszel method) [16]. The pooled OR was determined by $Z$-test, with significance level at $P<0.05$.

HWE was assessed using Fisher's exact test, with significance level at $P<0.05$. The funnel plot was used to estimate the potential publication bias. Egger's linear regression test on the natural logarithm scale of the OR was used to assess the funnel plot asymmetry, with significance level at $P<0.05$ [17]. STATA 11.0 was used to perform the statistical analyses (StataCorp, College Station, TX, USA). The "Venice criteria" were applied to each statistically significant association in the current meta-analysis to assess the credibility of the evidence [18].

\section{Results}

3.1. Studies and Populations. Nineteen studies were retrieved, among which six studies conformed to the inclusion criteria. Among the 13 excluded studies, five were reviews and five studies were not involved in Mink S38G gene polymorphism or AF. One study was excluded for being against the HWE. Two studies associated with Mink S38G gene polymorphism and AF were performed in non-Chinese population $[19,20]$. Following the 10-point scoring sheet by Clark and Baudouin, all selected studies achieved a score of no less than 8 [13]. The data were extracted from 856 cases and 1015 controls (Table 1) [7-9, 18-20].

3.2. Pooled Analyses. Mink S38G gene polymorphism was significantly related to AF under allelic (OR: 1.380, 95\% CI: 1.200-1.600, $P<0.00001$ ), recessive (OR: 1.193, 95\% CI: 1.033-1.377, $P=0.017$ ), dominant (OR: 1.057, 95\% CI: $1.025-$ 1.089, $P<0.00001$ ), additive (OR: 1.105 , 95\% CI: $1.036-1.178$, 
TABLE 3: The meta-regression results among 6 studies under the recessive genetic model for the association of Mink S38G gene polymorphism with $\mathrm{AF}$ in the Chinese population.

\begin{tabular}{lccccc}
\hline & Coefficient & Standard error & $T$ value & $P$ value & $95 \%$ confidence interval \\
\hline SS1 & 0.0883416 & 0.0103416 & 8.54 & $0.003^{*}$ & $0.05543 \sim 0.1212532$ \\
SG1 & -0.0245643 & 0.0028102 & -8.74 & $0.003^{*}$ & $-0.0335075 \sim-0.0156211$ \\
_cons & 0.678298 & 0.0669228 & 10.14 & $0.002^{*}$ & $0.4653198 \sim 0.8912762$ \\
\hline
\end{tabular}

${ }^{*} P<0.05$.

Coefficient: regression coefficient; the regression coefficients are the estimated increase in the lnOR per unit increase in the covariates; cons: constant item; SS1: SS genotype number of AF group sample size; SG1: SG genotype number of AF group.

TABLE 4: The meta-regression results among 6 studies under the additive genetic model for the association of Mink S38G gene polymorphism with $\mathrm{AF}$ in the Chinese population.

\begin{tabular}{lccccc}
\hline & Coefficient & Standard error & $T$ value & $P$ value & $95 \%$ confidence interval \\
\hline SS1 & 0.0387749 & 0.0047476 & 8.17 & $0.004^{*}$ & $0.0236658 \sim 0.0538841$ \\
SG1 & -0.0112773 & 0.0012901 & -8.74 & $0.003^{*}$ & $-0.015383 \sim-0.0071717$ \\
_cons & 0.3500916 & 0.030723 & 11.40 & $0.001^{*}$ & $0.2523172 \sim 0.4478659$ \\
\hline
\end{tabular}

${ }^{*} P<0.05$.

Coefficient: regression coefficient; the regression coefficients are the estimated increase in the lnOR per unit increase in the covariates; cons: constant item; SS1: SS genotype number of AF group sample size; SG1: SG genotype number of AF group sample size.

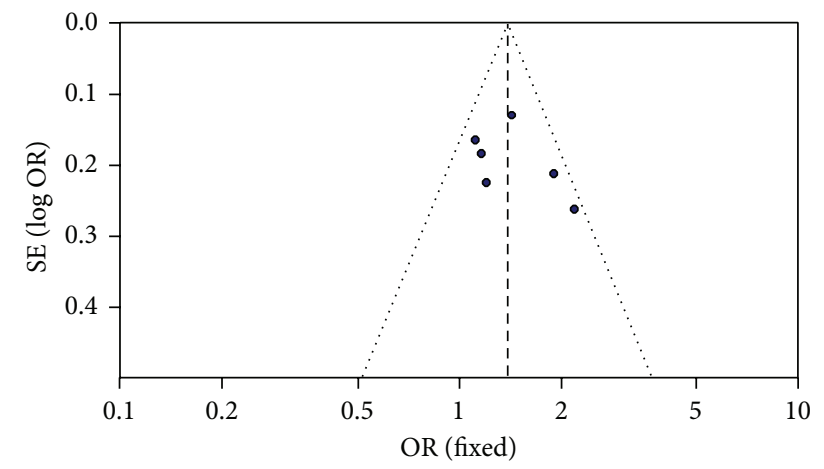

FIGURE 2: Funnel plot for studies of AF associated with Mink S38G gene polymorphism under an allelic genetic model (distribution of $\mathrm{G}$ allelic frequency of Mink S38G gene polymorphism) in the Chinese population. The horizontal and vertical axis correspond to the OR and confidence limits. OR: odds ratio; SE: standard error.

between the individual studies. Thus, significant publication bias existed in the present meta-analysis (allelic genetic model, $T=-3.83, P=0.019)$. The fail-safe number value was 40 under the additive genetic model which implied that 40 "null" studies should be added to render the general effect insignificant.

3.4. Sensitivity Analysis. A possible influence may be observed from the studies of Zeng et al. [8] and Qu and Yin [9]. Sensitivity analysis was conducted to assess the publication bias resource. After the two above studies were excluded, no obvious publication bias was shown in Egger's test (allelic genetic model, $T=-3.31, P=0.080$ ) and the funnel plot was approximately symmetrical (Figure 3 ).

\section{Discussion}

In the present meta-analysis, Mink S38G gene polymorphism was significantly related to AF under allelic (OR: 1.380),

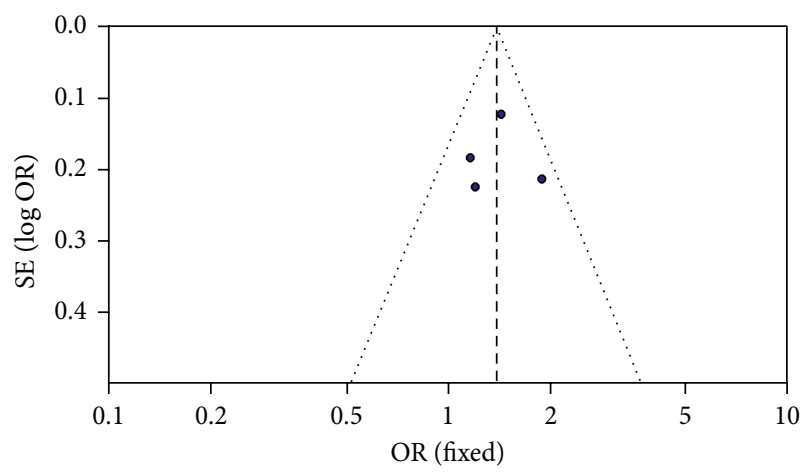

FIgURE 3: Funnel plot for studies of AF associated with Mink S38G gene polymorphism under an allelic genetic model (distribution of G allelic frequency of Mink S38G gene polymorphism) after sensitivity analysis in the Chinese population. The horizontal and vertical axis correspond to the OR and confidence limits. OR: odds ratio; SE: standard error.

recessive (OR: 1.193), dominant (OR: 1.057), additive (OR: 1.105), homozygous (OR: 1.128), and heterozygous genetic models (OR: 1.078) in the Chinese population. The current results suggested that the individuals with G allele of Mink S38G gene polymorphism may have higher AF susceptibility than the others in the Chinese population.

Heterogeneity existed under the recessive, additive, and multiplicative genetic models. Thus, subsequent metaregression was performed to explore the heterogeneity source. Under the recessive model, confounding factors such as SS1 $(P=0.003)$ and SG1 $(P=0.003)$ were demonstrated to possibly explain the heterogeneity source. After adjusting SG1, the AF risk only increased significantly in subgroup 2 which was not detected in subgroup 1. Heterogeneity disappeared in subgroup 2, but still existed in subgroup 1, suggesting that SG1 was the primary heterogeneity source. Similarly, under the additive model, SS1 $(P=0.004)$ and SG1 $(P=0.003)$ were the heterogeneity sources. In the subgroup 
analysis adjusted by SG1, the heterogeneity disappeared in subgroup 2 but remained in subgroup 1, indicating that SG1 was the main heterogeneity source.

In the present study, the significant associations of Mink S38G variants with AF were assessed using five genetic models, including allelic, recessive, dominant, additive, homozygous, and heterozygous genetic models. The $Z$-test was used to calculate the $P$ value; $P<0.05$ suggested a significant association. The fixed model was used under the allelic and dominant genetic models for the reason that their $P_{\text {heterogeneity }}>0.10$. Since the $P_{\text {heterogeneity }}<0.10$ under the recessive and additive genetic models, the random model was adopted.

The exclusion and inclusion criteria were addressed in detail and were strictly followed in the current meta-analysis. Based on the score system by Clark and Baudouin [13], all selected studies were with high quality. In the present meta-analysis, all individual studies were case-control studies published in official journals, where AF diagnostic was inconsistent with the major inclusion criteria. Family AF history has been excluded; thus, the meta-analysis results were objective and credible. Other unpublished studies that may contribute to the publication bias were not included in the current meta-analysis. Similarly, the relatively small sample size from the published studies also led to possible publication bias and heterogeneity among the individual studies. The sensitivity analysis showed that publication bias may come from the studies of Zeng et al. [8] and Qu and Yin [9]. After these studies were excluded, the publication bias disappeared. Based on the Venice criteria by Ioannidis et al., the epidemiology credibility was strong in all of the five genetic models [18].

$\mathrm{AF}$ is one of the most common clinical arrhythmias with complex pathogenesis. Recent studies showed that AF attack has a hereditary susceptibility. The genome-wide association studies (GWAS) identified three distinct genetic loci on chromosomes 1q21, 4q25, and 16q22 that are associated with AF. Susceptibility loci in 3p22, 5q35, 7q31, 12p12, and 12q24 were also identified by GWAS for PR interval duration. Only one locus around the gene NOS1AP was identified in the QT interval, another cardiovascular trait that is also a good indicator for AF [21].

At present, the gene variation encoding the ion channel was the pathologic factor for familial AF that reduced the Iks [22]. The Iks channel $\alpha$ and $\beta$ subunits were encoded by KCNQ1 and Mink genes, respectively [23]. On the physiological status, cardiac Iks channel participates in the atrial repolarization, especially on the terminal stage of action potential associated with frequency dependence action potential time interval shortening and atrial tissue electricity remodeling. Mink gene mutations can lead to various malignant arrhythmias such as familial long QT syndrome $\left(\mathrm{LQT}_{\mathrm{S}}\right)$ and Jervell syndrome $[24,25]$. Moreover, the Mink gene polymorphisms were associated with druginduced $\mathrm{LQT}_{\mathrm{S}}$ and nonfamilial arrhythmias [26]. Chevillard et al. (1993) found that the Mink gene mRNA expression increased distinctly in the atrial tissue of the AF patients [27]. Chen et al. (2003) found that the interaction of Mink protein with other proteins formed the arrhythmia matrix that can strike the onset or maintenance of AF [28]. Thus, the Mink gene is important in regulating cardiac rhythm. The possible reasons for the Mink 38G allele associated with AF were as follows. (a) Mink 38G allele could increase Mink gene expression and Iks channel protein synthesis. (b) Mink 38G allele could increase Iks current, thereby shorten the action potential duration, and be predisposed to $\mathrm{AF}$ [9].

Lin et al. (2009) performed a meta-analysis to explore Mink S38G gene polymorphism and AF and found that the GG genotype was significantly associated with increased AF risk [29]. However, their research was associated with some defects. First, two studies deviating from HWE were not excluded from their study [30,31]. Second, two studies published in 2008 were not included in their meta-analysis $[9,19]$. Compared with their work, the abovementioned four studies were appropriately addressed in the current meta-analysis. In addition, the study published in 2011 was also retrieved and supplemented in the present study [11]. Therefore, the current results should be more objective and credible than Zhang's work.

However, limitations still existed in the present metaanalysis. Large-scale studies on the association of AF with Mink S38G gene polymorphism were still relatively insufficient. The relatively large effect sizes in the current metaanalysis may be attributed to the limited sample size. The Mink expression level was influenced by the Mink S38G gene polymorphism and by other gene polymorphisms, such as KCNQ1 S140G, KCNE2 R27C, and KCNE3 R53H mutations.

Therefore, Mink S38G gene polymorphism was significantly associated with AF susceptibility. Patients with the G allele may be predisposed to AF. The current conclusion may guide the formulation of new individual AF diagnosis parameters and therapeutic strategies. Considering the abovementioned limitations, more large-scale studies are needed to confirm the above conclusion.

\section{Conflict of Interests}

The authors declare that there is no conflict of interests regarding the publication of this paper.

\section{Acknowledgments}

This work was funded by the National Natural Science Foundation of China (NSFC 81100073 to Dr. Yan-yan Li), Excellent Young and Middle-Aged Teachers Assistance Program of Nanjing Medical University for Dr. Yan-yan Li (2013-2015, JX2161015034), and the Priority Academic Program Development of Jiangsu Higher Education Institutions (PAPD). The authors would also like to thank all their colleagues working in the Department of Geriatrics, the First Affiliated Hospital of Nanjing Medical University.

\section{References}

[1] A. S. Go, E. M. Hylek, K. A. Phillips et al., "Prevalence of diagnosed atrial fibrillation in adults: national implications for rhythm management and stroke prevention: the anticoagulation and risk factors in atrial fibrillation (ATRIA) study," Journal of 
the American Medical Association, vol. 285, no. 18, pp. 23702375, 2001.

[2] G. Y. Lip, C. M. Brechin, and D. A. Lane, “The global burden of atrial fibrillation and stroke: a systematic review of the epidemiology of atrial fibrillation in regions outside north america and europe," Chest, vol. 142, no. 6, pp. 1489-1498, 2012.

[3] P. A. Wolf, R. D. Abbott, and W. B. Kannel, "Atrial fibrillation as an independent risk factor for stroke: the framingham study," Stroke, vol. 22, no. 8, pp. 983-988, 1991.

[4] R. Wachter, R. Lahno, B. Haase et al., "Natriuretic peptides for the detection of paroxysmal atrial fibrillation in patients with cerebral ischemia-the find-AF study," PLoS ONE, vol. 7, no. 4, Article ID e34351, 2012.

[5] S. Nattel, "New ideas about atrial fibrillation 50 years on," Nature, vol. 415, no. 6868, pp. 219-226, 2002.

[6] S. Nattel, "Atrial electrophysiological remodeling caused by rapid atrial activation: underlying mechanisms and clinical relevance to atrial fibrillation," Cardiovascular Research, vol. 42, no. 2, pp. 298-308, 1999.

[7] L.-P. Lai, M.-J. Su, H.-M. Yeh et al., "Association of the human minK gene $38 \mathrm{G}$ allele with atrial fibrillation: evidence of possible genetic control on the pathogenesis of atrial fibrillation," American Heart Journal, vol. 144, no. 3, pp. 485-490, 2002.

[8] Z.-Y. Zeng, J.-L. Pu, C. Tan et al., "The association of single nucleotide polymorphism of slow delayed rectifier $\mathrm{K}+$ channel genes with atrial fibrillation in Han nationality Chinese," Chinese Journal of Cardiovascular Diseases, vol. 33, no. 11, pp. 987991, 2005.

[9] Z. J. Qu and Y. H. Yin, "Correlations between Mink S38G polymorphism and atrial fibrillation in old patients with chronic heart failure," Chinese Journal of Biochemistry and Molecular Biology, vol. 24, pp. 1053-1057, 2008.

[10] A. Z. Ni, R. Wang, B. Liang, X. X. Zhu, J. Lin et al., "Relevance of gene KCNE1 and lone atrial fibrillation," Shanghai Medical Journal, vol. 27, pp. 260-261, 2004.

[11] L.-X. Xu, W.-Y. Yang, H.-Q. Zhang, Z.-H. Tao, and C.-C. Duan, "Study on the correlation between CETP TaqIB, KCNE1 S38G and eNOS T-786C gene polymorphisms for predisposition and non-valvular atrial fibrillation," Zhonghua Liuxingbingxue Zazhi, vol. 29, no. 5, pp. 486-492, 2008.

[12] J. Yao, Y.-T. Ma, X. Xie, F. Liu, B.-D. Chen, and Y. An, "Association of rs1805127 polymorphism of KCNE1 gene with atrial fibrillation in Uigur population of Xinjiang," Chinese Journal of Medical Genetics, vol. 28, no. 4, pp. 436-440, 2011.

[13] M. F. Clark and S. V. Baudouin, "A systematic review of the quality of genetic association studies in human sepsis," Intensive Care Medicine, vol. 32, no. 11, pp. 1706-1712, 2006.

[14] W. G. Cochran, "The effectiveness of adjustment by subclassification in removing bias in observational studies," Biometrics, vol. 24, no. 2, pp. 295-313, 1968.

[15] R. DerSimonian and N. Laird, "Meta-analysis in clinical trials," Controlled Clinical Trials, vol. 7, no. 3, pp. 177-188, 1986.

[16] N. Mantel and W. Haenszel, "Statistical aspects of the analysis of data from retrospective studies of disease," Journal of the National Cancer Institute, vol. 22, pp. 719-748, 1959.

[17] M. Egger, G. D. Smith, M. Schneider, and C. Minder, "Bias in meta-analysis detected by a simple, graphical test," British Medical Journal, vol. 315, no. 7109, pp. 629-634, 1997.

[18] J. P. A. Ioannidis, P. Boffetta, J. Little et al., "Assessment of cumulative evidence on genetic associations: Interim guidelines," International Journal of Epidemiology, vol. 37, no. 1, pp. 120-132, 2008.
[19] C. Fatini, E. Sticchi, M. Genuardi et al., "Analysis of $\min K$ and eNOS genes as candidate loci for predisposition to non-valvular atrial fibrillation," European Heart Journal, vol. 27, no. 14, pp. 1712-1718, 2006.

[20] A. Šmalcelj, J. Sertić, K. Golubić, L. Jurčić, L. Banfić, and M. Brida, "Interactions of MinK and e-NOS gene polymorphisms appear to be inconsistent predictors of atrial fibrillation propensity, but long alleles of ESR1 promoter TA repeat may be a promising marker," Collegium Antropologicum, vol. 33, no. 3, pp. 933-937, 2009.

[21] M. F. Sinner, P. T. Ellinor, T. Meitinger, E. J. Benjamin, and S. Kääb, "Genome-wide association studies of atrial fibrillation: past, present, and future," Cardiovascular Research, vol. 89, no. 4, pp. 701-709, 2011.

[22] Y. Yang, M. Xia, Q. Jin et al., "Identification of a KCNE2 gainof-function mutation in patients with familial atrial fibrillation," American Journal of Human Genetics, vol. 75, no. 5, pp. 899-905, 2004.

[23] J. Barhanin, F. Lesage, E. Guillemare, M. Fink, M. Lazdunski, and G. Romey, "K $\mathrm{K}_{\mathrm{v}} \mathrm{LQT1}$ and IsK (minK) proteins associate to form the $I_{\mathrm{KS}}$ cardiac potassium current," Nature, vol. 384, no. 6604, pp. 78-80, 1996.

[24] E. Schulze-Bahr, Q. Wang, H. Wedekind et al., "KCNE1 mutations cause jervell and Lange-Nielsen syndrome," Nature genetics, vol. 17, no. 3, pp. 267-268, 1997.

[25] I. Splawski, J. Shen, K. W. Timothy et al., "Spectrum of mutations in Long-QT Syndrome genes: KVLQT1, HERG, SCN5A, KCNE1, and KCNE2," Circulation, vol. 102, no. 10, pp. 1178-1185, 2000.

[26] A. D. C. Paulussen, R. A. H. J. Gilissen, M. Armstrong et al., "Genetic variations of HCNQ1, KCNH2, SCN5A, KCNE1, and KCNE2 in drug-induced long QT syndrome patients," Journal of Molecular Medicine, vol. 82, no. 3, pp. 182-188, 2004.

[27] C. Chevillard, B. Attali, F. Lesage et al., "Localization of a potassium channel gene (KCNE1) to 21q22.1-q22.2 by in situ hybridization and somatic cell hybridization," Genomics, vol. 15, no. 1, pp. 243-245, 1993.

[28] Y.-H. Chen, S.-J. Xu, S. Bendahhou et al., "KCNQ1 gain-offunction mutation in familial atrial fibrillation," Science, vol. 299, no. 5604, pp. 251-254, 2003.

[29] Z. Lin, Z. Li, L. Yafei, and M. Xiangyu, "MinK gene G112A polymorphisms and atrial fibrillation: a Meta-analysis," Journal of Medical Colleges of PLA, vol. 24, no. 4, pp. 198-207, 2009.

[30] A. Prystupa, G. Dzida, W. Myśliński, G. Małaj, and T. Lorenc, "MinK gene polymorphism in the pathogenesis of lone atrial fibrillation," Kardiologia Polska, vol. 64, no. 11, pp. 1205-1211, 2006.

[31] S. Lou, L. Lu, L. Q. Wu, W. F. Shen, JinQ et al., "Association between human potassium channel $\beta$-subunit gene KCNE1-S38G polymorphism and atrial fibrillation," Journal of Diagnostics Concepts \& Practice, vol. 5, pp. 415-418, 2006. 

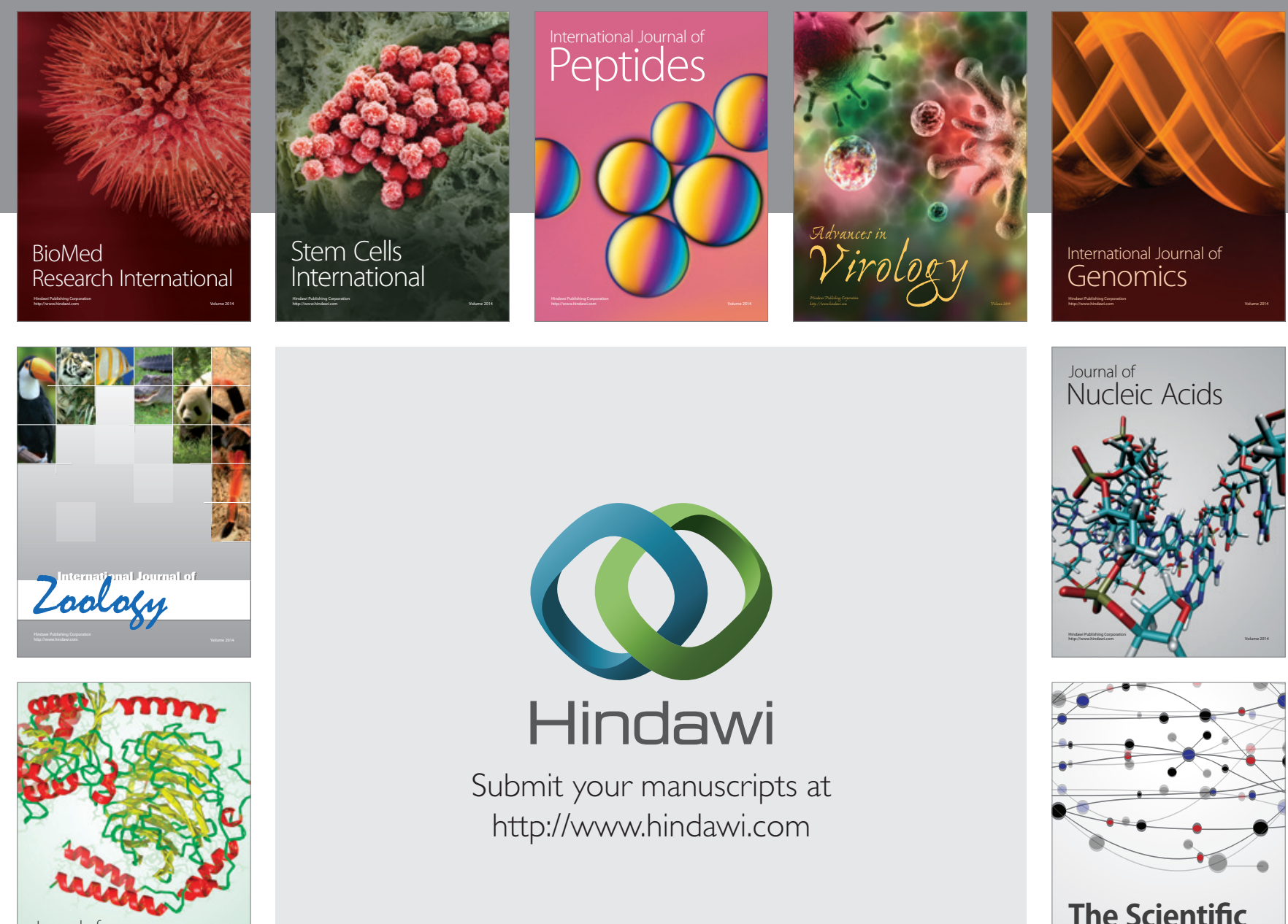

Submit your manuscripts at

http://www.hindawi.com

Journal of
Signal Transduction
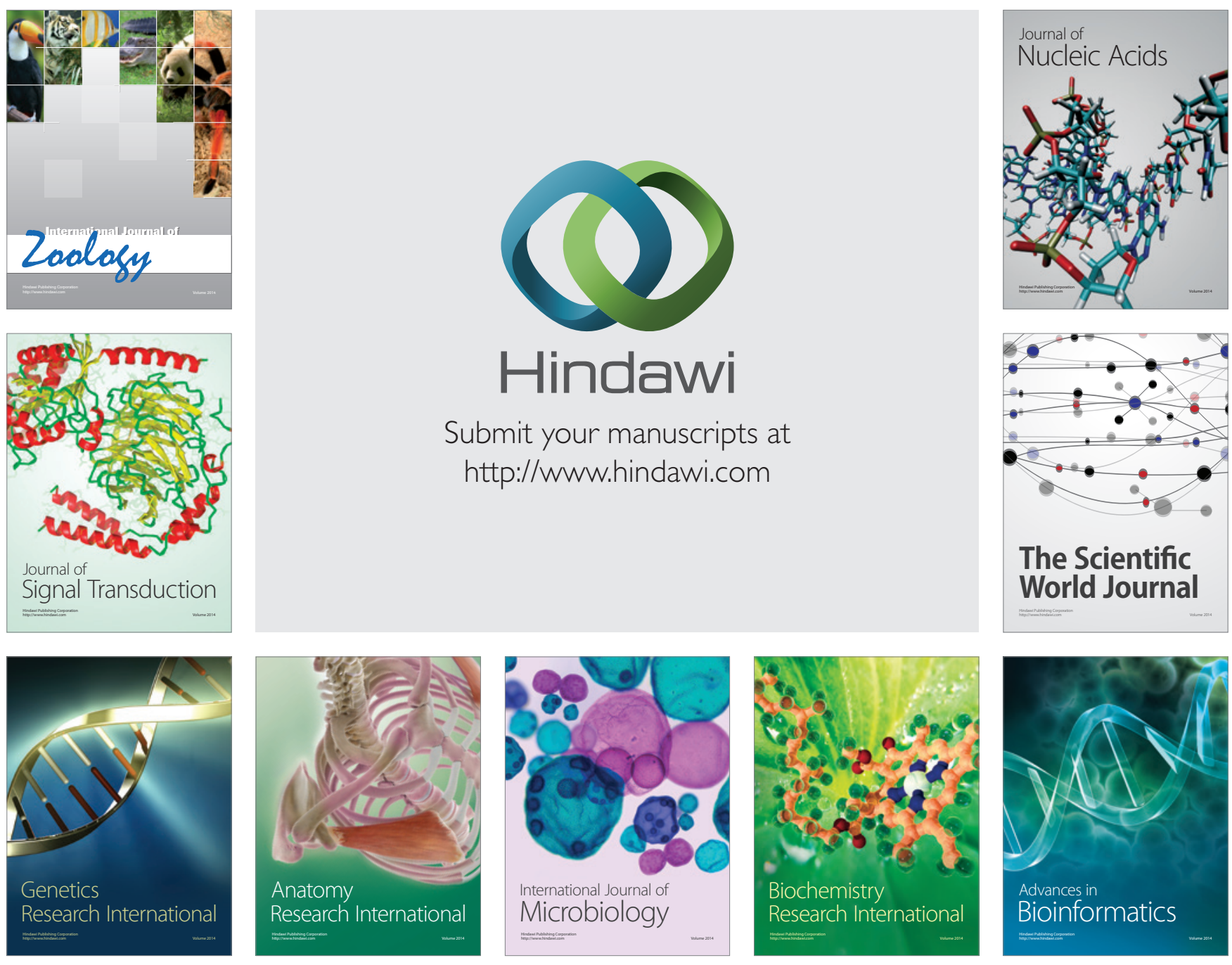

The Scientific World Journal
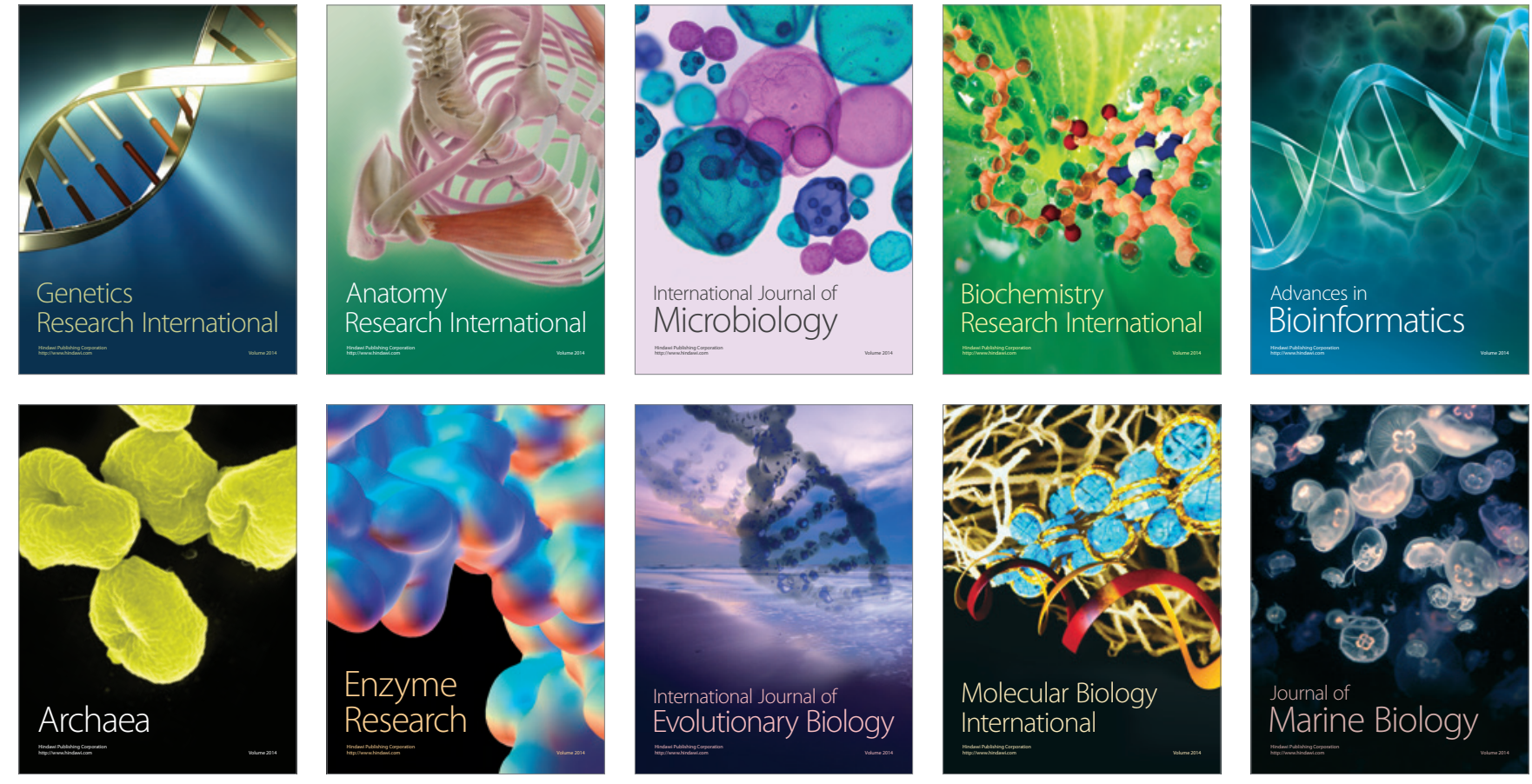